Supporting Information for

\title{
Might a 2,2-Dimethylbutane Molecule Serve as a Site to Promote Gas Hydrate Nucleation?
}

\author{
Zhengcai Zhang, †‡ Peter G. Kusalik,§ Guang-Jun Guo $^{\ddagger \| \perp *}$ \\ ${ }^{\dagger}$ Key Laboratory of Petroleum Resource Research, Institute of Geology and Geophysics, Chinese Academy of \\ Sciences, Beijing 100029, China. \\ ${ }^{\ddagger}$ Institutions of Earth Science, Chinese Academy of Sciences, Beijing 100029, China. \\ $\S$ Department of Chemistry, University of Calgary, 2500 University Drive NW, Calgary, T2N 1N4, Alberta, Canada. \\ "Key Laboratory of Earth and Planetary Physics, Institute of Geology and Geophysics, Chinese Academy of Sciences, \\ Beijing 100029, China. \\ ${ }^{\perp}$ College of Earth and Planetary Sciences, University of Chinese Academy of Sciences, Beijing 100049, China.
}

\section{AUTHOR INFORMATION}

Corresponding Author

*E-mail: guogj@mail.igcas.ac.cn 
Table S1. Force-field parameters used in this work.

\begin{tabular}{|c|c|c|c|c|}
\hline \multirow{3}{*}{ Water } & \multicolumn{4}{|c|}{ Geometric Parameters } \\
\hline & \multicolumn{2}{|c|}{ bond length (nm) } & \multicolumn{2}{|c|}{ angle (deg) } \\
\hline & $\mathrm{O}-\mathrm{H}$ & 0.9572 & $\mathrm{H}-\mathrm{O}-\mathrm{H}$ & 104.52 \\
\hline & $\mathrm{O}-\mathrm{M}$ & 0.1577 & & \\
\hline $\mathrm{CH}_{4}$ & $\mathrm{C}-\mathrm{H}$ & 1.0900 & $\mathrm{H}-\mathrm{C}-\mathrm{H}$ & 107.80 \\
\hline \multirow[t]{5}{*}{ DMB } & $\mathrm{C}-\mathrm{H}$ & 1.0900 & $\mathrm{H}-\mathrm{C}-\mathrm{H}$ & 107.80 \\
\hline & \multirow[t]{2}{*}{$\mathrm{C}-\mathrm{C}$} & \multirow[t]{2}{*}{1.5290} & C-C-C & 112.70 \\
\hline & & & C-C-H & 110.70 \\
\hline & \multicolumn{4}{|c|}{ Pair Potential Parameters } \\
\hline & & $\sigma(\AA)$ & $\varepsilon(\mathrm{kJ} / \mathrm{mol})$ & $q(\mathrm{e})$ \\
\hline \multirow[t]{3}{*}{ Water } & $\mathrm{O}$ & 3.1668 & 0.88217 & 0.0000 \\
\hline & $\mathrm{H}$ & 0.0000 & 0.00000 & 0.5897 \\
\hline & M & 0.0000 & 0.00000 & -1.1794 \\
\hline \multirow[t]{2}{*}{$\mathrm{CH}_{4}$} & $\mathrm{C}$ & 3.5000 & 0.27614 & -0.2400 \\
\hline & $\mathrm{H}$ & 2.5000 & 0.12552 & 0.0600 \\
\hline \multirow[t]{2}{*}{ DMB } & $\mathrm{C}$ & 3.5000 & 0.27614 & * \\
\hline & $\mathrm{H}$ & 2.5000 & 0.12552 & * \\
\hline
\end{tabular}

${ }^{*}$ The atomic charges for DMB were generated based on the $1.14^{*} \mathrm{CM} 1 \mathrm{~A}-\mathrm{LBCC}$ charge model, which is an optimized charge model for condensed-phase simulations, ${ }^{1}$ those can be generated from LigParGen website (http://zarbi.chem.yale.edu/ligpargen/). 


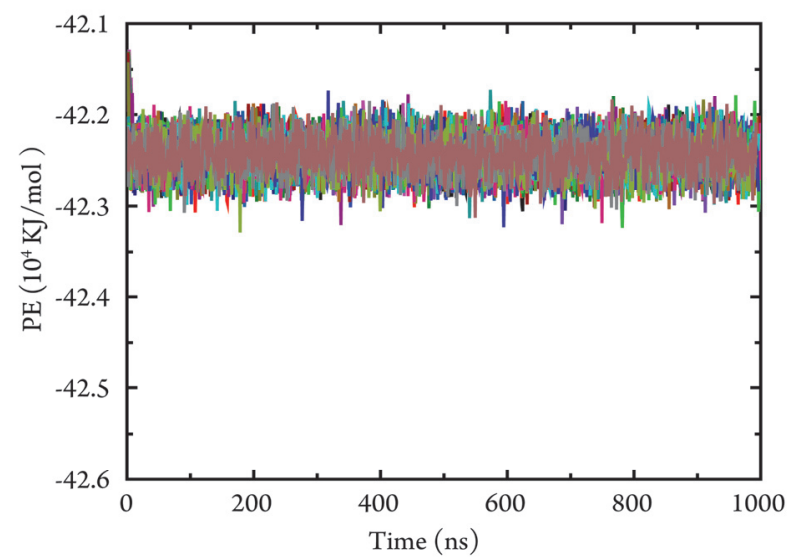

Figure S1. Time evolution of potential energy (PE) showing that no trajectories demonstrate hydrate nucleation events in system

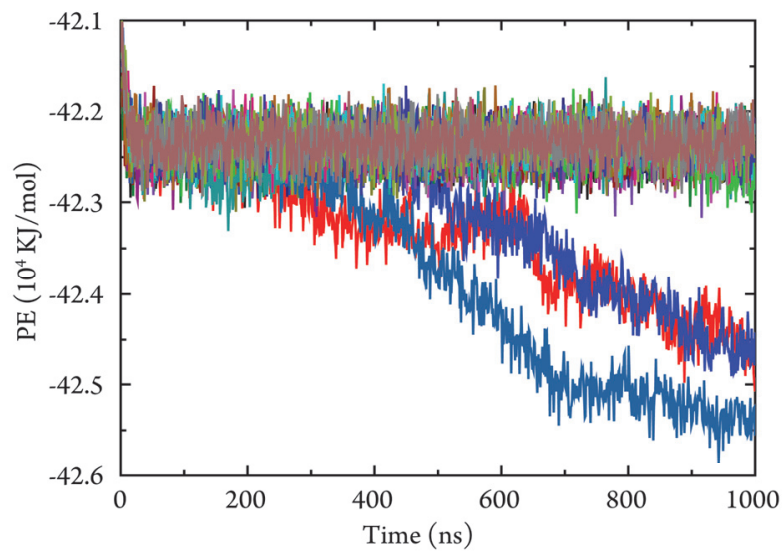

Figure S2. Time evolution of potential energy (PE) showing that four of twenty trajectories demonstrate hydrate nucleation events in system II. The green line shows that hydrate nucleates at $\sim 940 \mathrm{~ns}$.

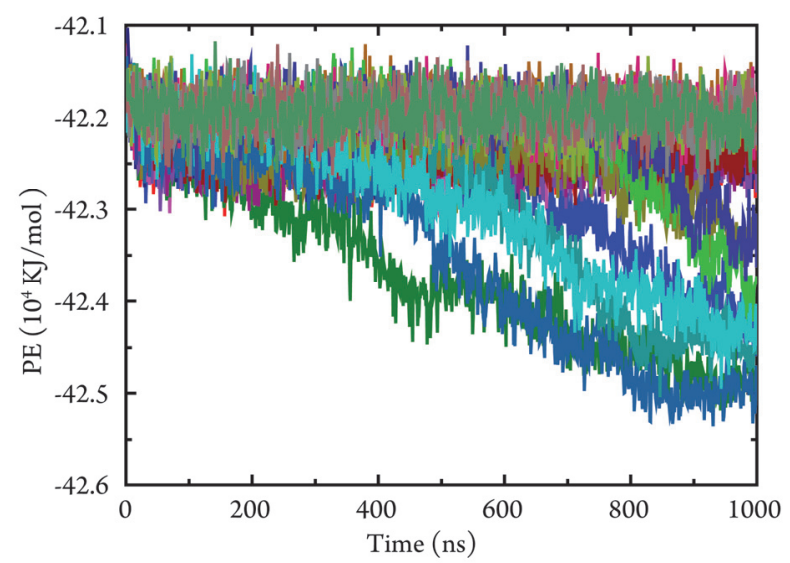

Figure S3. Time evolution of potential energy (PE) showing that eight of twenty trajectories demonstrate hydrate nucleation events in system III. 


\section{Definition of the first hydration shell and $\mathrm{CH}_{4}$ molecules associated with $\mathrm{DMB}$}

The first hydration shell of $\mathrm{CH}_{4}$ is defined as all the water molecules within a radius of $0.54 \mathrm{~nm}$ (dashed line in Figure $\left.\mathrm{S} 4(\mathrm{~A})\right)$ from the carbon atom in $\mathrm{CH}_{4}$. For DMB, the first hydration shell is defined as all the water molecules within the radius of $0.65 \mathrm{~nm}$ from the central carbon atom, labeled as green in the inset of Figure S4(B). The $\mathrm{CH}_{4}$ molecules associated with DMB are those at separations between 0.62 and $0.97 \mathrm{~nm}$ (as shown in Figure S4(C)) from the central carbon atom of DMB.
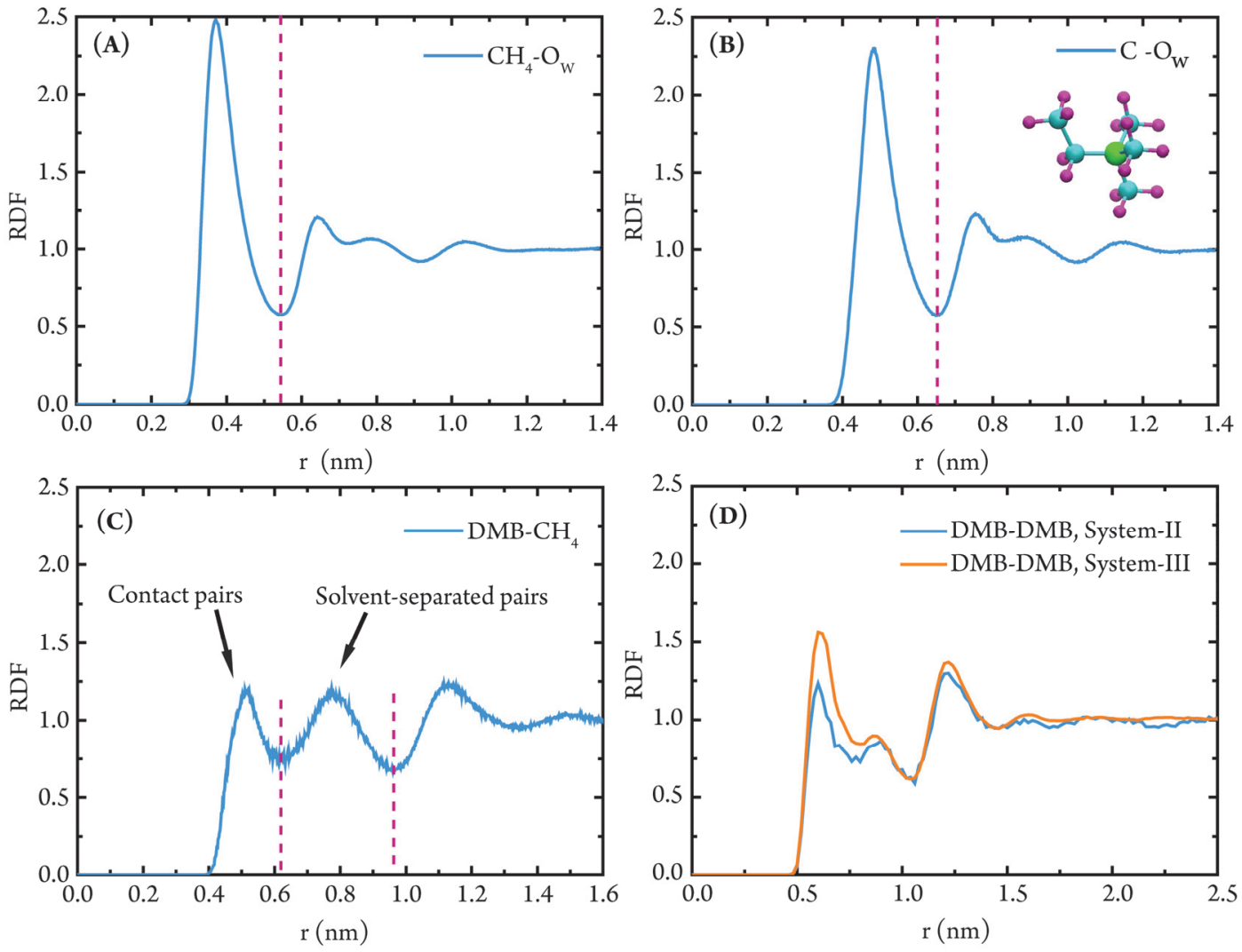

Figure S4. $\mathrm{CH}_{4}$-water (A), DMB-water (B), $\mathrm{DMB}_{-} \mathrm{CH}_{4}(\mathrm{C})$, and $\mathrm{DMB}-\mathrm{DMB}$ radial distribution functions $(\mathrm{RDF})$ calculated from a non-nucleating trajectory at $260 \mathrm{~K}$ and $10 \mathrm{MPa}$. The DMB-water RDF is between the oxygen atom in the water molecule and the central carbon atom (in green in the inset of $(B)$ ). The radius of the first hydration shell is delimited with the dashed lines in (A) and (B). 

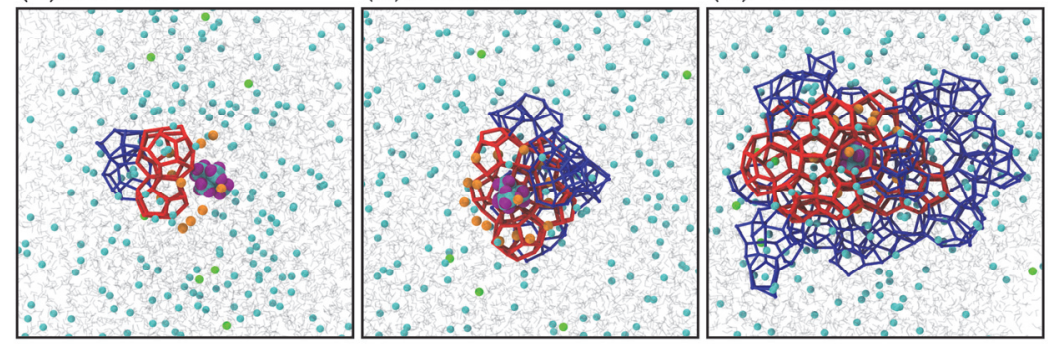

Figure S5. Snapshots from the trajectory in Figure 1 with face-saturated incomplete cages (FSICs) in the largest cluster shown (blue tubes). (A) is the same with Figure 1(D), where there is only one occupied FSIC. (B) is the same with Figure 1(G), where most of the FSICs are empty. (C) is the same with Figure $1(\mathrm{H})$, where FSICs are at the hydrate-aqueous interfacial region and most of them are empty, while CCs are in the inner part. The other color schemes are the same as that in Figure 1 . We find that there is a smaller number of FSICs at the very early stage of nucleation. As time evolves, FSICs continue to try to form but most of them are empty and fail to survive without the solutes present.

\section{Hydrate nucleation and growth processes in system III}

The time evolution of the potential energy and the number of complete cages (CC) in the largest cluster from a representative trajectory in system III is shown in Figure S6(A). It shows that the first persistent CC cluster forms at $\sim 282$ ns. However, after the cluster forms, the number of CCs in the cluster fluctuates around 4 for $\sim 140$ ns while the potential energy shows a slight drop as highlighted by the light green area in Figure S6(A). Snapshots detailing successive events of nucleation are shown in Figure S6(B to I) to illustrate the process. Before the occurrence of the persistent cage cluster, the first hydration shell of one DMB (shown in VDW representation in Figure S6(B to I)) is not fully ordered, yet several $\mathrm{CH}_{4}$ molecules(in orange) become associated with it (Figure S6 (B)). At 281.40ns, the first $5^{12}$ cage forms around one of these associated $\mathrm{CH}_{4}$ molecules. At 281.88 ns, another water cage forms adjacent to the first formed $5^{12}$ cage. While the number of cages in the CC cluster fluctuates, more cages are seen to form around the DMB (Figure S6(D to H)). At $358.86 \mathrm{~ns}$, the first hydration shell of the DMB is first seen to become a CC (Figure S6 (G)), although it never becomes a $5^{12} 6^{8}$ cage within our simulation time. The first standard $5^{12} 6^{8}$ cage forms at $543.56 \mathrm{~ns}$ and it is in the outermost layer of the CC cluster (a pink cage in the bottom-right corner of Figure S6 (I)) but it soon dissociates. After 425 ns, fast growth of the cage cluster is observed as can be seen in Figure S6 (A, H and I). It is noteworthy that during the time period of 282 to $425 \mathrm{~ns}$, although the number of cages in the largest CC cluster fluctuates around 4, the number of $\mathrm{CH}_{4} \mathrm{molecules}$ associated with the DMB first hydration shell actually increases (Figure S6 (B to I)). This is also shown in Figure S6 (J), where the number of $\mathrm{CH}_{4}$ molecules associated with the first hydration shell of DMB increases within the light green area. 

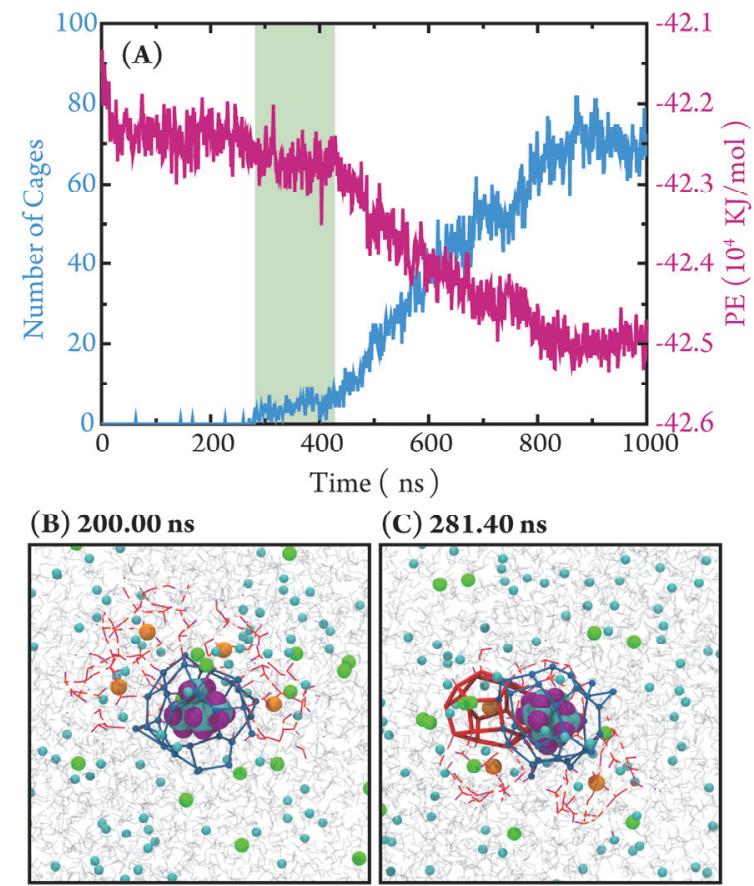

(D) $281.88 \mathrm{~ns}$

(E) $282.48 \mathrm{~ns}$
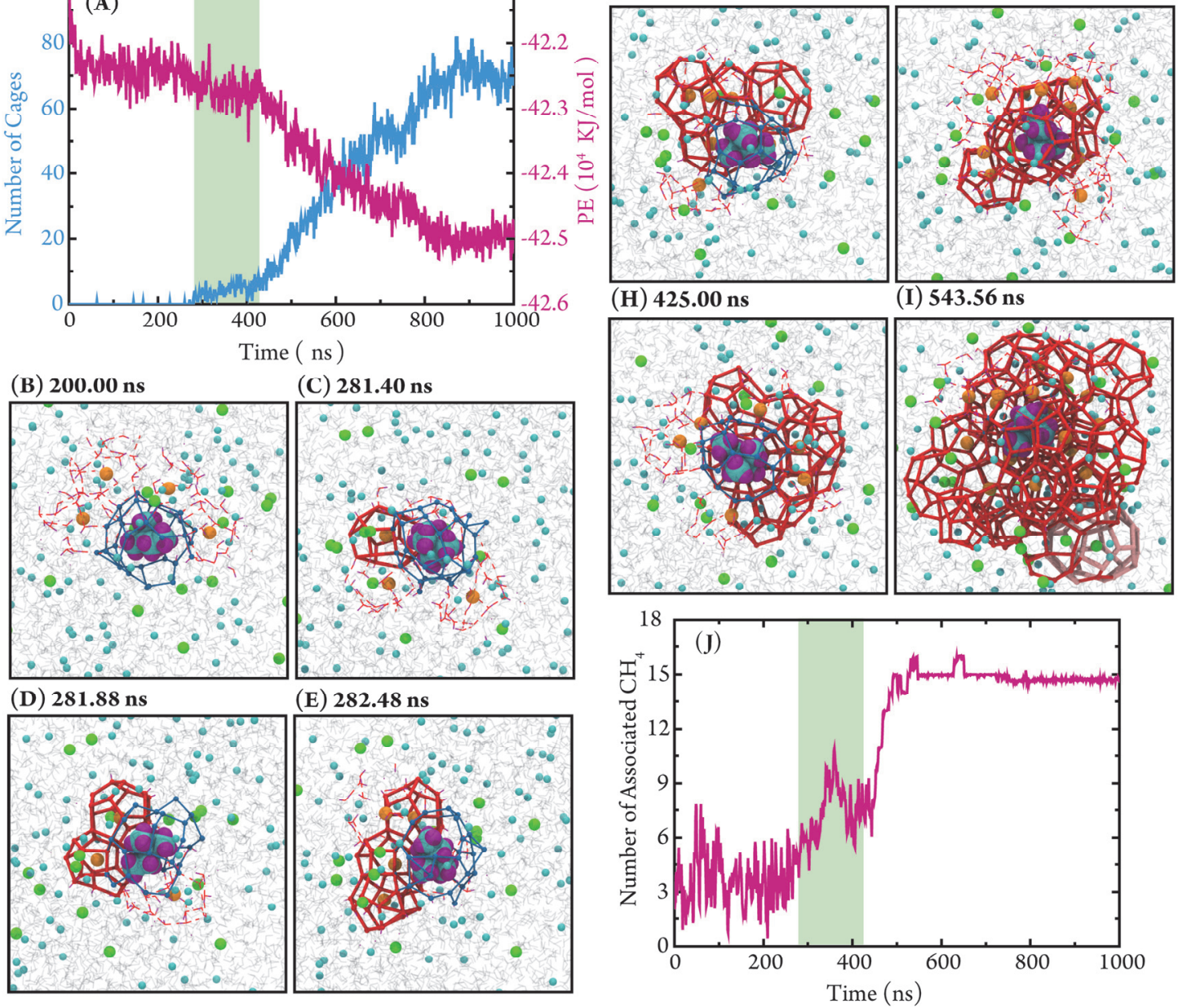

Figure S6. DMB acts as a $\mathrm{CH}_{4}$ hydrate promoter in system III. (A) The time evolution of the potential energy (PE) and the number of complete cages $(\mathrm{CC})^{2}$ in the largest CC cluster from a representative trajectory. Snapshots (B) through (I) show the CC cluster evolution from the trajectory in (A). The DMB acting as the hydrate promoter in this trajectory is shown in VDW representation (carbon in cyan, hydrogen in purple). The water molecules in its first hydration shell (first hydration shell) are represented as blue spheres and tubes, and the $\mathrm{CH}_{4}$ molecules associated with its first hydration shell are shown as orange spheres. The first hydration shell of the associated $\mathrm{CH}_{4}$ molecules is shown with red dashed lines. Other $\mathrm{DMB}$ and $\mathrm{CH}_{4}$ molecules are shown as green and cyan spheres, respectively. The cages in the largest CC cluster are shown as red tubes. The first standard $5^{12} 6^{8}$ cage is in pink in the bottom-right corner of (I). Other water molecules are represented as gray lines. $(\mathrm{J})$ The time evolution of the number of $\mathrm{CH}_{4}$ molecules associated with the first hydration shell of the highlighted DMB molecule. The light-green shadow area highlights the time period where the number of CCs fluctuates around 4 but the number of the associated $\mathrm{CH}_{4} \mathrm{molecules}$ on the first hydration shell of DMB increases. 


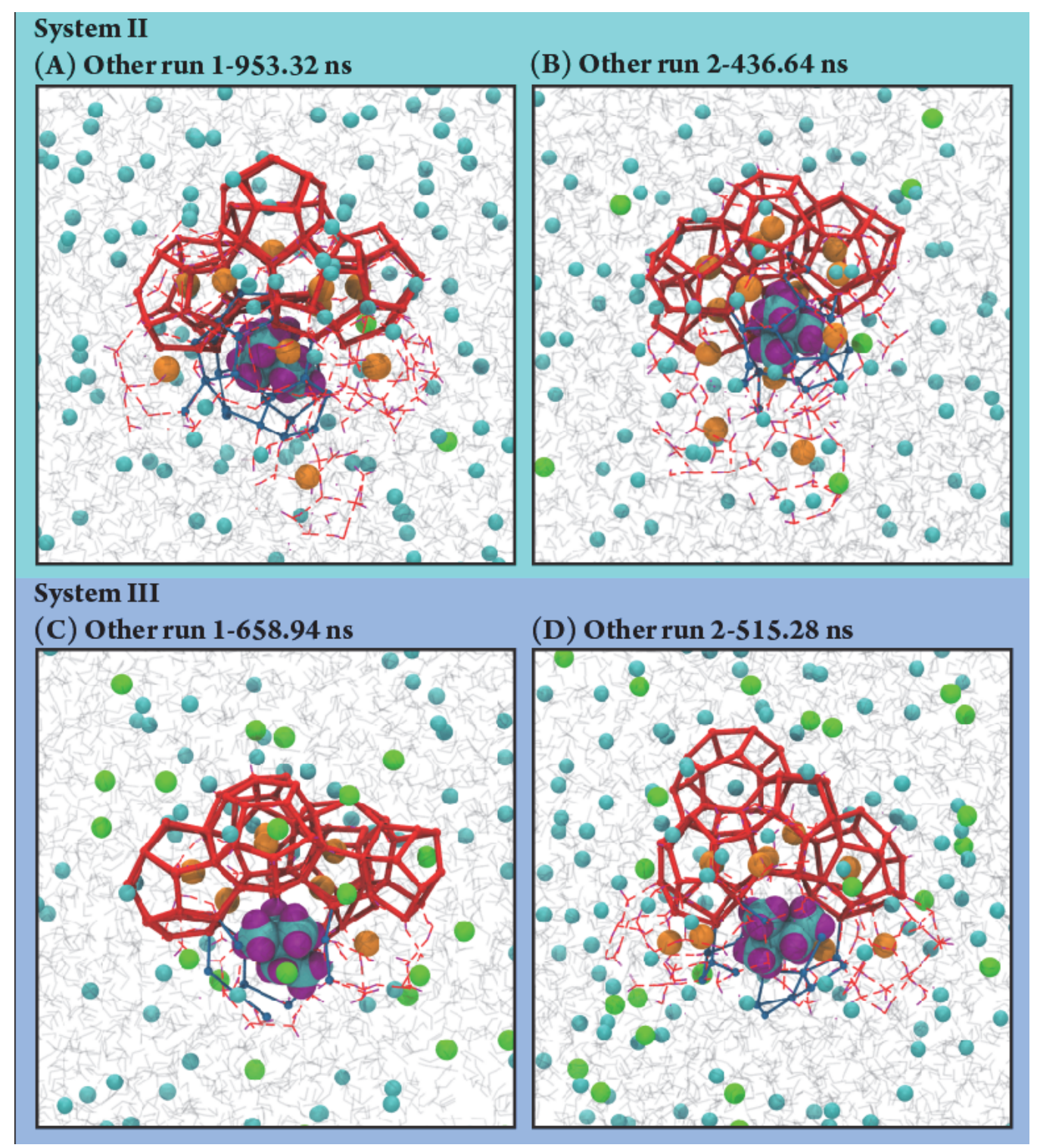

Figure S7. Snapshots similar to Figure 1(G) but from four other runs of systems II and system III. The color scheme is the same as that in Figure S6. 


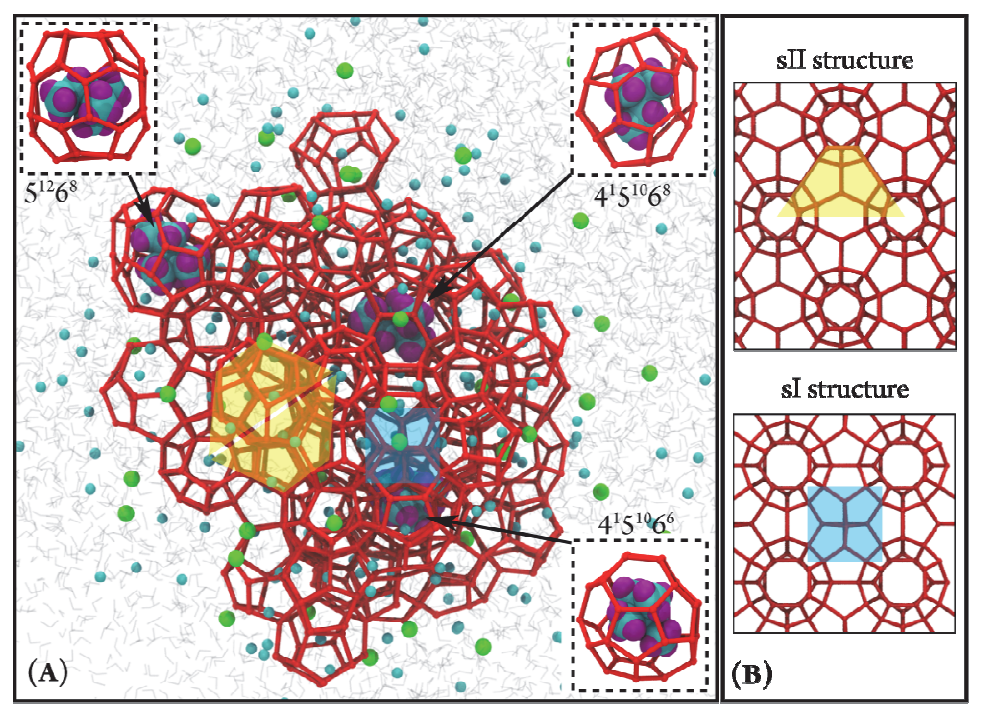

Figure S8. (A) The snapshot at the end of the simulation from Figure S6. The CC cluster contains thirty-nine $5^{12}$ cages, nine $5^{12} 6^{2}$ cages, three $5^{12} 6^{3}$ cages, three $5^{12} 6^{4}$ cages, one $5^{12} 6^{8}$ cage, nine $4^{1} 5^{10} 6^{2}$ cages, two $4^{1} 5^{10} 6^{3}$ cages, one $4^{1} 5^{10} 6^{4}$ cage, one $4^{1} 5^{10} 6^{6}$ cage, one $4^{1} 5^{10} 6^{8}$ cage, one $4^{2} 5^{8} 6^{1}$ cage, two $4^{2} 5^{8} 6^{2}$ cages, and two $4^{2} 5^{8} 6^{4}$ cages. (B) shows the crystalline structures of sI and sII hydrates. The color scheme is the same as that in Figure S6. The sI motifs is highlighted with a blue square, and the sII motif with a yellow trapezoid. The DMB filled cages are highlighted in (A). To help sharpen the structure of the cluster, the end configuration has been quenched at $2 \mathrm{~K}$.
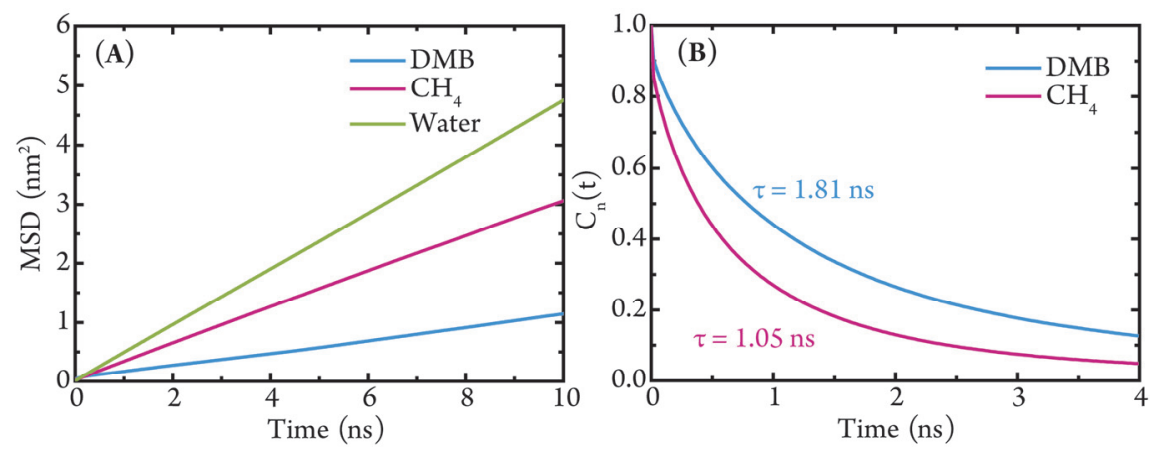

Figure S9. Dynamical properties of the molecules in system III from a non-nucleating trajectory. (A) The mean-square displacement (MSD) of molecules in system. (B) Residence autocorrelation function, $\mathrm{C}_{\mathrm{n}}(t){ }^{3}$ of water molecules in the first hydration shell of DMB and $\mathrm{CH}_{4} \cdot \mathrm{C}_{\mathrm{n}}(t)=<n(0) n(t)>/\langle n(0) n(0)>$, where $n(t)=1$ if the water molecules stay in the first hydration shell without departing at any time between time $t=0$ and $t$. By fitting with $C_{n}(t)$ to the form $A e^{-t / \tau}$, where $A$ is a pre-exponential factor, we can obtain the residence time of the first hydration shell, $\tau$, as shown in(B). 

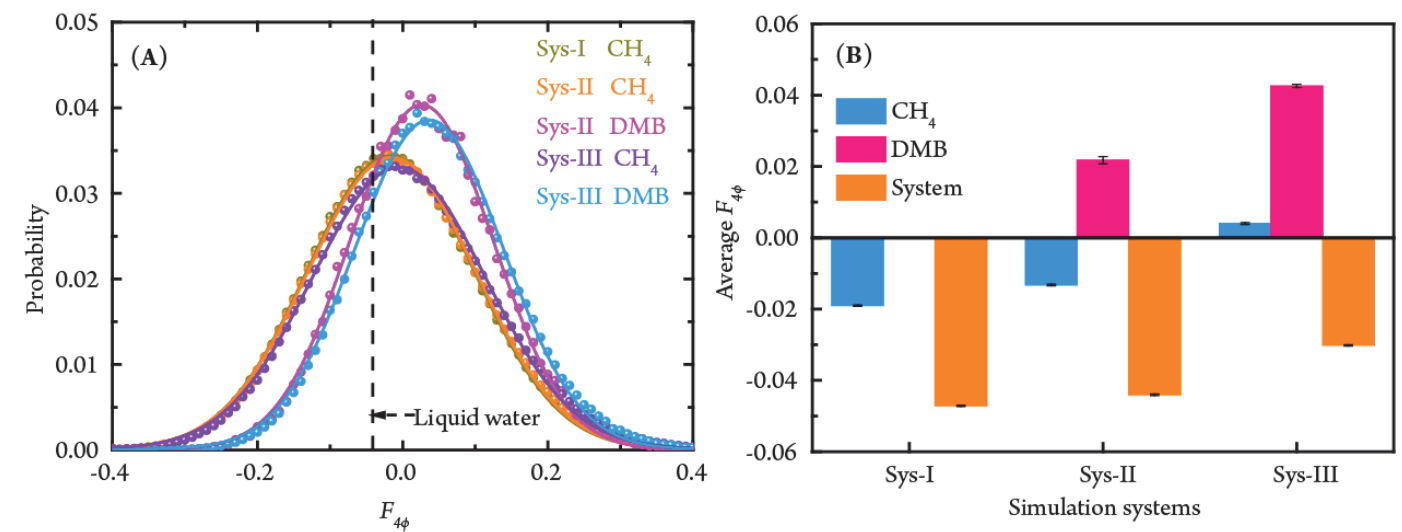

Figure S10. (A) The probability distribution of the average $F_{4 \varphi}$ of water molecules within the first hydration shell of DMB and $\mathrm{CH}_{4}$ for all the systems used in this study, and (B) the average $\mathrm{F}_{4 \varphi}$ for all the first hydration shells of DMB and $\mathrm{CH}_{4}$ and for all water molecules in the system. The $F_{4 \varphi}=\langle\cos (3 \varphi)>$, where $\varphi$ is the torsion angle between two hydrogen-bonded water molecules. The values for ice, liquid water, and hydrate is $-0.4,-0.04$, and 0.7 , respectively. ${ }^{4}$ The results are from non-nucleating trajectories. One can find that the first hydration shell of $\mathrm{DMB}$ is more hydrate-like than that of $\mathrm{CH}_{4}$ (i.e. has larger $\mathrm{F}_{4 \varphi}$ values). With increasing DMB concentration (from system I to system III), the first hydration shells of both DMB and $\mathrm{CH}_{4}$ become somewhat more hydrate-like.

Table S2. The nucleation time of $\mathrm{CH}_{4}$ hydrate nucleation at our working conditions. The nucleation time, $t^{*}$, is estimated as :

\begin{tabular}{|c|c|c|c|c|}
\hline \multicolumn{5}{|c|}{ number of nucleating trajectories } \\
\hline System & $x_{\mathrm{CH} 4}$ & $n_{\mathrm{nu}} / n_{\mathrm{run}}$ & $\begin{array}{c}\text { Induction time (ns) } \\
\text { (for nucleating trajectories) }\end{array}$ & $t^{*}(\mathrm{~ns})$ \\
\hline I & 0.039 & $0 / 20$ & - & $>20000$ \\
\hline I' & 0.05 & $7 / 10$ & $106 ; 643 ; 328 ; 281 ; 706 ; 639 ; 182$ & 841 \\
\hline$I^{\prime \prime}$ & 0.06 & $10 / 10$ & $96 ; 91 ; 44 ; 126 ; 126 ; 49 ; 105 ; 48 ; 94 ; 109$ & 89 \\
\hline II & 0.038 & $4 / 20$ & $436 ; 165 ; 272 ; 938$ & 4500 \\
\hline III & 0.033 & $8 / 20$ & $356 ; 193 ; 656 ; 837 ; 459 ; 702 ; 242 ; 790$ & 2000 \\
\hline
\end{tabular}

\section{Estimation of the nucleation rate in system I}

Using data for $\mathrm{CH}_{4}$ concentrations $\left(x_{\mathrm{CH} 4}\right)$ and nucleation times $\left(t^{*}\right)$ of $\mathrm{CH}_{4}$ hydrate reported by Lauricella et al. ${ }^{52}$ with a similar model system at $250 \mathrm{~K}$, we find a good fit to exponential behavior (see Figure S11). Consequently, we performed two additional sets of simulations with systems the same as the system I but with mole fractions of $\mathrm{CH}_{4}$ of 0.05 and 0.06 . Using estimates of the average nucleation time of $\mathrm{CH}_{4}$ hydrate from these two additional sets of simulations (I' and I" in Table S2), and assuming that the nucleation time (rate) has an exponential dependence on the $\mathrm{CH}_{4}$ concentration, we estimate a nucleation time of $12 \pm 7 \mu$ s for system I. 


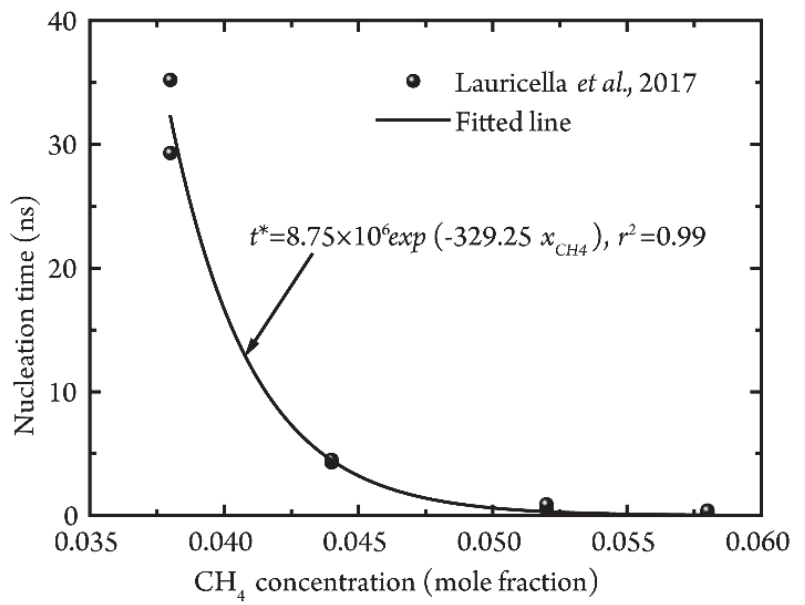

Figure S11. The plot of the $\mathrm{CH}_{4}$ concentration dependence of nucleation time at $250 \mathrm{~K}$ and $50 \mathrm{MPa}$ from Lauricella et al. ${ }^{5}$ The exponential form $t^{*}=a \exp \left(-b x_{\mathrm{CH} 4}\right)$ fits the data well. Note that the volume of all systems remains almost constant.

Movie S1. The movie shows that DMB acts as a $\mathrm{CH}_{4}$ hydrate promoter in system III from one of the trajectories in Figure $\mathrm{S} 3$ (time period 100-500 ns). The color scheme is the same as that of Figure S6. 


\section{References}

1. Dodda, L. S.; Vilseck, J. Z.; Tirado-Rives, J.; Jorgensen, W. L., 1.14*CM1A-LBCC: Localized Bond-Charge Corrected CM1A Charges for Condensed-Phase Simulations. J. Phys. Chem. B, 2017, 121, 3864-3870.

2. Guo, G.-J.; Zhang, Y.-G.; Liu, C.-J.; Li, K.-H., Using the Face-Saturated Incomplete Cage Analysis to Quantify the Cage Compositions and Cage Linking Structures of Amorphous Phase Hydrates. Phys. Chem. Chem.Phys. 2011, 13, 12048-12057.

3. Allen, M. P.; Tildesley, D. J., Computer Simulation of Liquids; Clarendon Press, 1989, p. 385.

4. Rodger, P. M.; Forester, T. R.; Smith, W., Simulations of the Methane Hydrate Methane Gas Interface near Hydrate Forming Conditions. Fluid Phase Equilibria 1996, 116, 326-332.

5. Lauricella, M.; Ciccotti, G.; English, N. J.; Peters, B.; Meloni, S., Mechanisms and Nucleation Rate of Methane Hydrate by Dynamical Nonequilibrium Molecular Dynamics. J. Phys. Chem. C 2017, 121, 24223-24234. 